\title{
The effect of curing temperature and time on the acoustic and optical properties of PVCP
}

\author{
Marina Bakaric, Student Member, IEEE, Piero Miloro, Bajram Zeqiri, Ben T. Cox, \\ and Bradley E. Treeby, Member, IEEE
}

\begin{abstract}
Polyvinyl chloride plastisol (PVCP) has been increasingly used as a phantom material for photoacoustic and ultrasound imaging. As one of the most useful polymeric materials for industrial applications, its mechanical properties and behaviour are well-known. Although the acoustic and optical properties of several formulations have previously been investigated, it is still unknown how these are affected by varying the fabrication method. Here, an improved and straightforward fabrication method is presented and the effect of curing temperature and curing time on PVCP acoustic and optical properties, as well as their stability over time, is investigated. Speed of sound and attenuation were determined over a frequency range from 2 to $15 \mathrm{MHz}$, while the optical attenuation spectra of samples was measured over a wavelength range from 500 to 2200 nm. Results indicate that the optimum properties are achieved at curing temperatures between $160^{\circ} \mathrm{C}$ and $180^{\circ} \mathrm{C}$, while the required curing time decreases with increasing temperature. The properties of the fabricated phantoms were highly repeatable, meaning the phantoms are not sensitive to the manufacturing conditions provided the curing temperature and time are within the range of complete gelation-fusion (samples are optically clear) and below the limit of thermal degradation (indicated by the yellowish appearance of the sample). The samples' long term stability was assessed over 16 weeks and no significant change was observed in the measured acoustic and optical properties.
\end{abstract}

Index Terms-Acoustic properties, optical properties, phantoms, photoacoustics, polyvinyl chloride plastisol, ultrasound.

\section{INTRODUCTION}

\section{A. Overview}

$\mathbf{P}$ OLYVINYL CHLORIDE PLASTISOL (PVCP) is a suspension of PVC particles in a liquid plasticizer. It is widely available for purchase as a two-part suspension of polyvinyl chloride resin (PVC and copolymers) and plasticizer that keeps the resin in a liquid state when at room temperature and forms a plastisol [1]. Upon mechanical mixing and heating between $70^{\circ} \mathrm{C}$ and $200^{\circ} \mathrm{C}$ (depending on the formulation), the plastic particles dissolve and the mixture turns into a gel of high viscosity. During the process of gelation, the plasticizer penetrates into the PVC grains, swelling them and glueing them together thus decreasing the relative amount of free plasticizer and forming a homogeneous material [2]. The rate of the gelation process is inversely proportional to the PVC molecular weight and particle size [3]. The gel strength

M. Bakaric, B.T. Cox and B.E. Treeby are with the Department of Medical Physics and Biomedical Engineering, University College London, London WC1E 6BT, U.K. (e-mail: marina.bakaric.16@ucl.ac.uk)

P. Miloro and B. Zeqiri are with the National Physical Laboratory, Teddington TW11 0LW, U.K.

This work was supported in part by the Engineering and Physical Sciences Research Council, grant numbers EP/L020262/1 and EP/P008860/1. increases with curing temperature, and the time required for gel formation decreases with increasing curing temperature. Just above the temperature of gelation, granular morphology is no longer observable and the material obtains optimum tensile properties in a process called fusion [4]. On cooling below $60^{\circ} \mathrm{C}$, a flexible, permanently plasticized solid material with elastomeric properties is formed [5].

As an industrial material, PVCP is well-characterised in terms of its mechanical properties and has well-established fabrication methods. Various PVC-based formulations are used with numerous additives that modify the properties and reduce the final cost of the produced articles [6]. However, these formulations are often not widely available for use in fabrication of tissue-mimicking phantoms and can be expensive, especially if purchased for small production batches. Commercial premixed PVC-plasticizer suspensions can be purchased at low cost as a phthalate-free plastisol base material commonly used for making fishing lures. As this application does not pose any strict requirements on the final product such as homogeneity and repeatability, several preparation methods for PVCP tissue-mimicking phantoms have been proposed utilizing standard laboratory equipment [7]-[11]. However, these studies have yielded different properties and considerations regarding the suitability of PVCP for applications in photoacoustic and ultrasound imaging. In this paper, the properties and preparation methods for PVCP phantoms are reviewed. A straightforward fabrication method is then introduced and the effect of fabrication temperature and curing time on the acoustic and optical properties of the material is investigated, and their stability over time is presented.

\section{B. Industrial PVCP - Mechanical Properties}

The effect of curing temperature and time on PVCP's mechanical properties (but not acoustic) has already been thoroughly investigated within the scope of its industrial application [12]. Curing conditions commonly used in industry are temperatures ranging from 160 to $220^{\circ} \mathrm{C}$ and curing times between 6 and 16 minutes. The optimum properties are obtained at 200 and $220^{\circ} \mathrm{C}$ for curing times of 14 and 10 minutes, respectively [12]. The tensile strength increases with curing temperature and time, while the elongation at break point is highly sensitive to thermal degradation [13] and is related to plasticizer structure and molecular weight, which is in turn directly linked to chain mobility (high molecular weight results in lower mobility and thus low elongation at break values). The effect of curing temperature and time can also be 
observed morphologically through microscopic characterisation, as well as by studying the colour and opacity of the cured samples which become transparent and acquire a colouration representative of the natural colour of the plasticizer used when fully cured [12]. A direct relationship between plasticizer migration and curing temperature and time is known: for lower degrees of curing, the plasticizer is not fully absorbed by the PVC particles and is thus free to migrate through the microstructure. The minimum values of migration are obtained at the same curing conditions as the optimum mechanical properties of the samples.

\section{Review of Phantom Fabrication Methods}

PVCP was first used as a tissue-mimicking material for ultrasound elastography in 2003 [14]. Although no description was given on how the phantoms were prepared, the work provided valuable information on the speed of sound and acoustic attenuation of the material, indicating its tissue-like properties and utility.

The first reported process for fabrication of PVCP as a phantom for photoacoustic imaging consisted of heating a commercially available plastisol formulation (manufacturer not reported) [7]. The heating was done under partial vacuum in order to eliminate air bubbles. The liquid PVCP was then allowed to cool, reheated and poured into a mould of the desired shape containing various targets. The use of titanium dioxide $\left(\mathrm{TiO}_{2}\right)$ to introduce optical scattering and commercial plastic colours in order to improve absorption was suggested.

The acoustic and optical properties of PVCP were first measured in [8]. The material was also characterized as a potentially suitable tissue-mimicking phantom for use in biomedical photoacoustics as it is non-toxic, insoluble in water and can be reused without degradation. The phantoms were prepared by heating a mixture of liquid PVCP and BPC (Black Plastic Colour, CI Pigment Black 7) to approximately $200{ }^{\circ} \mathrm{C}$, stirred continuously with a magnetic stirrer. As it is heated, the opaque PVCP-BPC mixture becomes more viscous and translucent which is when $\mathrm{TiO}_{2}$ powder was added. The total heating time reported for $300 \mathrm{ml}$ of mixture was $130 \mathrm{~min}$. Upon cooling, the PVCP mixture solidifies and can easily be removed from glass containers and cut into the desired shape. The properties are summarised in Table I. The reheating of PVCP was reported to alter its optical properties. The speed of sound was insensitive to the addition of colourants during phantom fabrication but did introduce scattering and increase optical absorption. The provider of the materials was M-F Manufacturing Co. (Fort Worth, TX, USA), which is commonly used in all studies reviewed to date, unless stated otherwise. This study reports that the phantoms should be stored away from other plastic materials as PVCP can react with and dissolve other plastics when in prolonged contact through mutual diffusion. A multi-layered PVCP skin phantom [15] has been made using this preparation procedure [8].

The characterisation of PVCP mixtures ranging from super soft to super rigid enabled their use for fabrication of a realistic prostate phantom [9]. Phantoms of varying degrees of softness were made by adding $25 \%$ to $90 \%$ softener agent (a plasticizer additive that reduces hardness and increases flexibility), yielding speed of sound values from $1580 \mathrm{~m} \cdot \mathrm{s}^{-1}$ to $1360 \mathrm{~m} \cdot \mathrm{s}^{-1}$, respectively, thus concluding that the speed of sound of PVCP can be tuned by the addition of the hardener/softener agent. It should be noted, however, that the acoustic attenuation was not measured. The mixtures were made using a hot plate set to $450{ }^{\circ} \mathrm{C}$ (no information was given on the temperature reached within the PVCP suspension), heated for 20 to 30 minutes and then placed into a vacuum chamber for 1 to 2 minutes prior to pouring into an aluminium mould. The mould was preheated to prevent the PVCP from curing too quickly around the walls which was known to trap the escaping air bubbles. The fabricated PVCP phantoms were left to cool down and were stored in glass containers [15], stored at $-21^{\circ} \mathrm{C}$.

In 2013, two new fabrication processes were developed. The first one consisted of degassing liquid PVCP suspension at room temperature and then pouring it into the mould and heating in an oven at either $130^{\circ} \mathrm{C}$ or $170^{\circ} \mathrm{C}$ for plastic and super soft plastic, respectively, for two hours [10]. The phantom properties were tuned by adding PVC and/or graphite powder. The authors reported that the curing temperature changed all material properties except the speed of sound, but this was not further investigated. The acoustic properties of two formulations can be found in Table I. The phantoms were also characterised thermally, yielding a specific heat capacity between 1.16 and $2.65 \mathrm{~J} \cdot \mathrm{kg}^{-1} \cdot \mathrm{K}^{-1}$ and thermal conductivity $\kappa$ from 0.0603 to $0.1243 \mathrm{~J} \cdot \mathrm{s}^{-1} \cdot \mathrm{K}^{-1} \cdot \mathrm{m}^{-1}$. The speed of sound was shown to decrease linearly by $20 \%$ in the temperature range from $20^{\circ} \mathrm{C}$ to $50{ }^{\circ} \mathrm{C}$. Although making the fabrication process easy, this method had several drawbacks such as bubble formation during heating and graphite particle settling. The properties of PVCP with $2 \%$ graphite powder in the temperature range from $20^{\circ} \mathrm{C}$ to $45^{\circ} \mathrm{C}$ were later measured [16] and two phantoms were made: soft $\left(\rho=953 \pm 24 \mathrm{~kg} \cdot \mathrm{m}^{-3}\right)$ and hard $\left(\rho=972 \pm 24 \mathrm{~kg} \cdot \mathrm{m}^{-3}\right)$ with a specific heat capacity of $2.65 \pm 0.51 \mathrm{~J} \cdot \mathrm{kg}^{-1} \cdot \mathrm{K}^{-1}$ and thermal conductivity of 0.091 $\pm 0.013 \mathrm{~J} \cdot \mathrm{s}^{-1} \cdot \mathrm{K}^{-1} \cdot \mathrm{m}^{-1}$. The speed of sound decreased from $1501.5 \pm 0.7 \mathrm{~m} \cdot \mathrm{s}^{-1}$ at $22{ }^{\circ} \mathrm{C}$ to $1331.8 \pm 0.3 \mathrm{~m} \cdot \mathrm{s}^{-1}$ at $45^{\circ} \mathrm{C}$, while the acoustic attenuation coefficient changed from 0.46 $\pm 0.03 \mathrm{~dB} \cdot \mathrm{cm}^{-1}$ to $0.94 \pm 0.09 \mathrm{~dB} \cdot \mathrm{cm}^{-1}$ at the frequency of $1 \mathrm{MHz}$. The fabrication method still had issues with graphite settling.

The second PVCP fabrication protocol was developed for tuning its optical properties to values relevant for studies of biological tissue in the near infrared [11]. The aim of this work was to obtain a standard physical phantom for routine quality control and performance evaluation of photoacoustic imaging instruments. The protocol, called $\mathrm{S} 1$, is as follows: upon adding the optical absorbers (BPC) and scatterers $\left(\mathrm{TiO}_{2}\right)$ to liquid PVCP suspension at room temperature, the suspension is sonicated at $40^{\circ} \mathrm{C}$ for 10 minutes; then poured into a flask along with a magnetic stirrer bar, placed in an oil bath preheated to $200{ }^{\circ} \mathrm{C}$ and connected to a vacuum line which is gradually opened until small bubbles are observed in the mixture. During the procedure, the vacuum level should be adjusted to avoid excessive bubbling. After approximately 6 minutes, the material should reach $130{ }^{\circ} \mathrm{C}$ and become viscous, so the stirring speed should be reduced. After 2 additional minutes, the material should begin to move freely so stirring 
should be restored back to its original speed. After 10 minutes, the stirrer bar should be moving freely and the material should have reached $180^{\circ} \mathrm{C}$, when it is ready for pouring into a mould. Several phantoms were made in the study with varying concentrations of BPC (0 to $0.016 \% \mathrm{v} / \mathrm{v}$ for background and 0.064 to $0.256 \% \mathrm{v} / \mathrm{v}$ for targets, yielding optical absorption coefficients of 0 to $0.1 \mathrm{~cm}^{-1}$ and 0.26 to $1.07 \mathrm{~cm}^{-1}$, respectively) and $\mathrm{TiO}_{2}$ in concentrations of 0 to $2.5 \mathrm{mg} \cdot \mathrm{ml}^{-1}$ for the targets yielding a reduced optical scattering coefficient of 0.9 to 6.8 $\mathrm{cm}^{-1}$. The addition of $\mathrm{TiO}_{2}$ was reported to have no effect on the acoustic attenuation coefficient values. The phantoms were stored in air-tight containers and showed no sign of physical degradation, nor bacterial growth during the course of six months. This would make PVCP a suitable candidate for a standard photoacoustic phantom, however, the material did not satisfy other requirements such as the ease of preparation and mimicking the ultrasound properties of tissues.

In another study, PVCP's suitability as a potential phantom for photoacoustic vascular imaging was considered due to its stability and longevity [17]. The effect of PVC concentration and the addition of biologically relevant absorbers and scatterers on its acoustic and optical properties was investigated. The phantoms were made following protocol S1 [11]. Hardener agent was added (0 to $50 \% \mathrm{v} / \mathrm{v}$ ) in order to increase PVC concentration, while $2 \mathrm{mg} \cdot \mathrm{ml}^{-1}$ to 0.125 $\mathrm{mg} \cdot \mathrm{ml}^{-1}$ of $\mathrm{TiO}_{2}$ and $1 \% \mathrm{v} / \mathrm{v}$ to $0.125 \% \mathrm{v} / \mathrm{v}$ BPC were added to tune the reduced scattering coefficient $\mu_{s}^{\prime}$ and optical absorption coefficient $\mu_{a}$, respectively. The optical properties were measured in the wavelength range from $500 \mathrm{~nm}$ to 1100 $\mathrm{nm}$ and the ability of PVCP to be tuned to tissue-relevant properties was demonstrated. $\mu_{s}^{\prime}$ of the PVCP suspension was reported to be less than $0.05 \mathrm{~cm}^{-1}$ making it a good base material, while $\mu_{a}$ exhibited absorption peaks characteristic of the primary plasticizer bis(2-ethylhexyl) adipate (DEHA), which were reduced with the addition of BPC. The acoustic attenuation coefficient increased from approximately 3 to 5 $\mathrm{dB} \cdot \mathrm{cm}^{-1}$ at the frequency of $5 \mathrm{MHz}$ with increasing hardener concentration from $0 \%$ to $50 \%$, however the speed of sound of PVCP was effectively unalterable. The addition of $\mathrm{TiO}_{2}$ had no effect on acoustic properties. The requirement for adequate tunability of the acoustic properties established the ground for further work in which a dual-plasticizer approach for fabricating PVCP phantoms with finely-tunable acoustic and optical properties was developed [18]. The plasticizer choice had a great impact on the speed of sound and acoustic attenuation of PVCP and four of the most suitable formulations were used for fabrication, with PVC resin content of 10 to $20 \%$. A positive correlation between the speed of sound and plasticizer density was observed, but none with molecular weight. Glass microspheres were used to tune the acoustic attenuation and ultrasound speckle pattern. The best storage conditions for PVCP phantoms were also thoroughly investigated: encased in a solid chamber and sealed to prevent environmental exposure, then stored at a low temperature of $2{ }^{\circ} \mathrm{C}$ to reduce plasticizer exudation.

The suitability of PVCP as a quantitative photoacoustic imaging (qPAI) phantom has also been assessed [19]. The suspension used was Lure Flex Firm provided by Lure Fac- tors (Doncaster, UK), a British equivalent to the previously mentioned M-F Manufacturing Co. PVCP was characterized over the wavelength range from $400 \mathrm{~nm}$ to $2000 \mathrm{~nm}$ and intrinsic absorption peaks were observed at 910, 1190, 1400 and $1720 \mathrm{~nm}$, possibly due to vibrational energy transitions in PVC [20]. The use of various pigments and sulphates as chromophores for multiwavelength photoacoustic imaging phantoms has been thoroughly investigated [21]. Nonlinearity of the optical absorption coefficient was observed at high peak powers typically used in photoacoustic imaging. The Grüneisen parameter was measured to be $\Gamma=1.01 \pm 0.05$.

In another study, the mechanical and acoustic properties of PVCP were also altered by adding softener, mineral oil and glass microspheres in order to develop a regression model for the design of formulations with targeted properties [22]. The optical clarity of samples was represented by light transmittance at two wavelengths (645 nm and $532 \mathrm{~nm}$ ) and increased with the addition of softener, while it decreased with the addition of microspheres and oil, the latter being due to the impediment of cross-linking. The elastic modulus, Shore hardness and viscoelastic relaxation time constant exhibited a wide range of tunability, whereas the speed of sound and acoustic attenuation had a large discrepancy with values of real soft tissues. The regression model was thus applied to mechanical properties only and validated experimentally with an error $<5 \%$ within the range of previously measured parameters.

A comprehensive list of acoustic and optical properties of PVCP phantoms fabricated and characterised in the above studies and the values obtained in this study is given in Table I.

\section{Paper Outline}

Using the existing methods, it is challenging to produce repeatable phantoms with consistent properties, thus PVCP is not the favoured choice for a tissue-mimicking material, in spite of its longevity, stability and robustness. In this paper, an optimized protocol for the fabrication of PVCP phantoms using a commercially available PVC-plasticizer suspension is presented. The effect of curing temperature and time on the acoustic and optical properties of PVCP is then investigated. The fabrication method used is described in Section II, along with the methods employed to characterise the samples. Acoustic and optical properties of fabricated PVCP samples are presented in Section III, and their stability over time is reported.

\section{Materials And Methods}

\section{A. Phantom Fabrication}

Taking into account the reviewed fabrication methods, the PVCP samples were initially made following protocol S1 [11] and using Lure Flex Firm provided by Lure Factors, Doncaster, UK. The following observations were made. Firstly, the movement of the magnetic stirrer bar was soon disabled as the surrounding suspension solidifies more quickly. Secondly, it is considered that the temperature/time recommendations from the protocol [11] may have been inaccurately reported. 
TABLE I

SPEED OF SOUND $c$, DENSITY $\rho$, ACOUSTIC ATTENUATION COEFFICIENT $\alpha$ AND OPTICAL ABSORPTION COEFFICIENT $\mu_{a}$ FOR VARIOUS PVCP FORMULATIONS FROM THE LITERATURE.

\begin{tabular}{|c|c|c|c|c|c|}
\hline Formulation & Ref. & $\mathrm{c}\left(10^{3} \mathrm{~m} \cdot \mathrm{s}^{-1}\right)$ & $\rho\left(10^{3} \mathrm{~kg} \cdot \mathrm{m}^{-3}\right)$ & $\alpha\left(\mathrm{dB} \cdot \mathrm{cm}^{-1}\right)$ & $\mu_{a}\left(\mathrm{~cm}^{-1}\right)$ \\
\hline Triple hardness PVCP & {$[14]$} & 1.395 & - & $1.05 @ 4.5 \mathrm{MHz}$ & - \\
\hline PVCP (M-F Manufacturing) & [8] & $1.40 @ 1 \mathrm{MHz}$ & $0.98-1.01$ & $0.57 @ 1 \mathrm{MHz}$ & $0-3.7 @ 1064$ nm \\
\hline PVCP with $25 \%$ softener & [9] & 1.58 & 0.98 & - & - \\
\hline PVCP with $50 \%$ softener & [9] & 1.44 & 0.91 & - & - \\
\hline PVCP with $75 \%$ softener & [9] & 1.42 & 0.93 & - & - \\
\hline PVCP with $90 \%$ softener & [9] & 1.36 & 0.74 & - & - \\
\hline PVCP cured at $130^{\circ} \mathrm{C}$ & [10] & 1.43 & 0.97 & $0.66 @ 1 \mathrm{MHz}$ & - \\
\hline PVCP cured at $170^{\circ} \mathrm{C}$ & [10] & 1.43 & 0.97 & $0.92 @ 1 \mathrm{MHz}$ & - \\
\hline PVCP Protocol S1 & [11] & - & - & - & $0-1.07 @ 600-1000$ nm \\
\hline Dual-plasticizer formulation & [18] & $1.35-1.54$ & - & $1-30 @ 4.0 \mathrm{MHz}$ & $0.07-10 @ 500-1100 \mathrm{~nm}$ \\
\hline PVCP with $5-20 \%$ softener & [19] & $1.40 @ 5 \mathrm{MHz}$ & 1.01 & $6.45-8.12 @ 5 \mathrm{MHz}$ & $0-3 @ 400-2000 \mathrm{~nm}$ \\
\hline PVCP at investigated curing conditions & Current study & $1.40 @ 5 \mathrm{MHz}$ & $0.95-1.07$ & 7.26-8.24@5 MHz & $0.45-16 @ 500-2200$ nm \\
\hline
\end{tabular}

The suspension temperature was monitored using a suspended thermometer and exhibited a slow temperature rise, reaching $130{ }^{\circ} \mathrm{C}$ after 30 minutes, as opposed to the reported $180{ }^{\circ} \mathrm{C}$ which could not be achieved within a reasonable amount of time (a maximum of 6 hours was tested). It was noticed that if the thermometer was touching the bottom of the flask, a higher temperature occurred within the amount of time reported, but this meant the actual readings were taken of the Duran glass and not the suspension. The saturation temperature of the suspension using the oil bath setup was $150^{\circ} \mathrm{C}$. This temperature depended very little on the concentration of softener used. Nonetheless, the protocol proved to give optically inconsistent phantoms (acoustic properties were not investigated at this point). Preventing bubble formation or premature solidifying during pouring into the mould was also a significant challenge. These difficulties encountered while following the fabrication protocol S1 may be related to the composition of the PVCplasticizer suspension used, as the effect of the particle size and distribution on PVCP viscoelasticity is a well reported phenomenon [23].

The fabrication method suggested by the provider of the PVCP suspension utilizes a microwave oven. However, following this method caused the PVCP to carbonise even at the lowest microwave power level. It was thus concluded that making PVCP should involve a gradual heating process.

Finally, a method was adopted following the main guidelines from [10] with additional steps to prevent bubble formation. The fabrication process was as follows:

1) PVCP suspension was mechanically mixed (by shaking for approximately 5 minutes) with any softener/hardener agent or pigment dispersion additives.

2) The mixture was then sonicated using an ultrasonic bath (T9, L\&R Manufacturing Co., Kearny, NJ, USA) with the heater set to $40^{\circ} \mathrm{C}$ for 10 minutes in order to improve component mixing.

3) The mixture was poured into Anumbra ${ }^{\circledR}$ resistance glass petri dishes $(\varnothing 50 \mathrm{~mm})$ used as moulds and degassed for 10 minutes in a vacuum chamber at 0.4 mbar to eliminate bubbles created during mixing. The sample thicknesses were around $5 \mathrm{~mm}$ and $10 \mathrm{~mm}$.

4) The dishes were placed in an oven (ED 23, Binder, Tuttlingen, Germany) preheated to $40{ }^{\circ} \mathrm{C}$ and the temperature gradually elevated to the desired set temperature (average heat up time of the oven from $40{ }^{\circ} \mathrm{C}$ to the set temperature was 20 minutes) after which the mixtures were left to cure over the chosen amount of time.

5) Upon curing, the disk-shaped samples were left to cool at room temperature before demoulding and stored in glass containers at $2{ }^{\circ} \mathrm{C}$.

In order to investigate the effect of curing temperature and time on PVCP properties, first the minimum and maximum curing temperatures were established. The maximum time of 2 hours was chosen in order to keep the fabrication process convenient. The curing timing was started at the point when the oven reached the set temperature. Gradual heating of the PVCP suspension is desirable as placing the samples at high temperatures causes layered solidification and results in non-uniform samples. The minimum temperature required for solidification of PVCP was $120^{\circ} \mathrm{C}$, while samples started to burn at $220^{\circ} \mathrm{C}$. Thus 14 samples were made at temperatures ranging from $120^{\circ} \mathrm{C}$ to $200^{\circ} \mathrm{C}$ in steps of $20^{\circ} \mathrm{C}$, and curing times of $15,30,60$ and 120 minutes. For temperatures below $180^{\circ} \mathrm{C}$ the minimum time required for solidification was 30 minutes, while the samples at $200^{\circ} \mathrm{C}$ were only cured for 15 minutes as longer times were not achievable because the fabrication conditions constituted a fire hazard due to oven limitations and laboratory safety conditions.

Several phantoms were also made using pigment dispersions as additives. Various colours were used in different concentrations $(0.01 \%, 0.1 \%$ and $1 \%)$ obtained by serial dilution of pigment with PVCP suspension. All pigments were supplied by the same manufacturer as for the PVCP suspension. The coloured samples were cured at $160^{\circ} \mathrm{C}$ for 120 minutes. Cylindrical phantoms with volumes up to $\approx 200 \mathrm{~mL}$ have also been made using the same fabrication protocol, although there should be no inherent limit on the size of the phantom.

\section{B. Optical Characterisation}

PVCP optical properties were characterized using a dual-beam spectrophotometer (Lambda 750, Perkin Elmer, Waltham, MA, USA). PVCP disks were placed on sample mounts and collimated-transmission absorbance measurements were made at four different locations on each sample (two per side) over the wavelength range from $500 \mathrm{~nm}$ to $2200 \mathrm{~nm}$. The total optical attenuation arises from the combined effects 
of absorption and scattering. In the absence of scattering, the optical absorption coefficient could be calculated from these measurements using the sample thickness values taken from the acoustic measurements described below. A qualitative assessment of the optical transparency of PVCP (the degree of scattering) was also made by placing samples over a mesh pattern and observing their clarity.

\section{Acoustic Characterisation}

The density of the phantoms was determined using the buoyancy method in accordance with BS EN 1183-1:2019. The precision balance used for these measurements was ML203T/00 (Mettler Toledo, Columbus, OH, USA). The density was further used to calculate the acoustic impedance by multiplying the density and the speed of sound of the phantom.

Acoustic properties of the samples were characterized using a broadband through-transmission setup [24] available in the NPL Materials Measurement Facility (National Physical Laboratory, Teddington, UK). Speed of sound and transmission loss were measured using a substitution technique with reference to water [25]. A broadband transducer with centre frequency 10 $\mathrm{MHz}$ and $10 \mathrm{~mm}$ active diameter (Force Technology, Brøndby, Denmark) was used as the emitter and a $30 \mathrm{~mm}$ active element diameter broadband bilaminar hydrophone manufactured by Marconi (Essex, UK) was used as a receiver. Thickness was also simultaneously evaluated using the reflected signals from the front and rear surface of the samples. Attenuation was obtained from transmission loss data by using samples of two different thicknesses [26] of around $5 \mathrm{~mm}$ and $10 \mathrm{~mm}$. The temperature during the measurements was $20^{\circ} \mathrm{C} \pm 0.5^{\circ} \mathrm{C}$, measured using a calibrated spirit-in-glass thermometer (IP 39C, G. H. Zeal, London, UK).

The measurement uncertainty was evaluated following the guide to the expression of uncertainty and confidence in measurement [27]. The expanded measurement uncertainty quoted in the results section was determined using both Type A (random) and Type B (systematic) uncertainty evaluations and is given as the standard uncertainty multiplied by a coverage factor, $k=2$, providing a coverage probability of approximately $95 \%(\mathrm{p}=0.95)$, according to the method recommended in [27], [28]. Systematic uncertainties arise from several sources which were independently evaluated at different frequencies. Some of these include the temperature variations, diffraction errors, oscilloscope resolution and the linearity of the amplifier and the receiver [26], [28].

\section{Stability Characterisation}

In order to evaluate the phantoms' stability over time, the per cent mass and density loss, as well as the acoustic and optical properties were measured 16 weeks after fabrication. Initial characterisation measurements were done within $72 \mathrm{~h}$ of fabrication. Density was included as a parameter as this made the inter-sample comparison more clear.

\section{RESUlTS AND DiscUSSION}

\section{A. Optical Properties}

Fig. 1 shows a comparison of the colour and opacity of PVCP samples fabricated with different curing temperatures

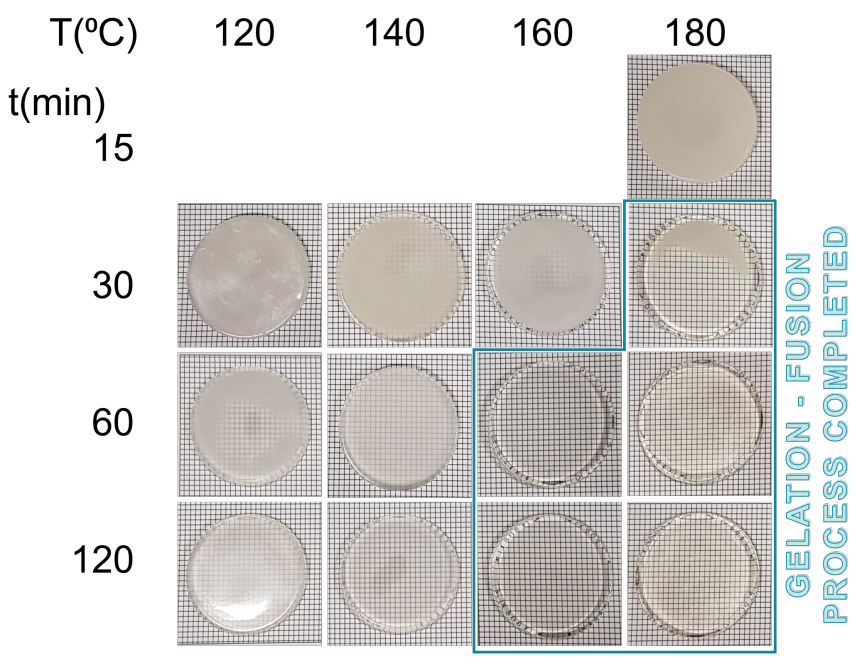

Fig. 1. Colour and transparency of polyvinyl chloride plastisol (PVCP) assessed by placing the samples over a mesh pattern. (Colour in online version.)

and times. The partially cured samples (incomplete gelationfusion) appear white and are opaque and inhomogeneous, while the fully cured samples (complete gelation-fusion) are optically clear. This is a good method of testing whether the fabrication process was successfully completed. It is concluded that the optimal fabrication temperature lies above $160{ }^{\circ} \mathrm{C}$ and the required curing time decreases with increasing temperature. As the fabrication method does not include mixing during heating, a shorter curing time is desirable in order to reduce the settling of particles when scatterers are added. Consequently, the recommended curing conditions are a temperature of $180^{\circ} \mathrm{C}$ and curing time of 30 minutes. The samples heated to $200{ }^{\circ} \mathrm{C}$ with a curing time of 15 minutes exhibited a yellow appearance, thus indicating thermal degradation [13]. In industry, the colouration of PVC formulations is commonly used as an indication of the grade of thermal degradation of the samples [29]. It is a consequence of the polymer dehydrochlorination and leads to changes in crosslinking, poor mechanical properties and potential stability issues [30]. Thus these samples were exempt from further investigation.

The grade of completeness of the gelation-fusion process is also indicated by the characteristic optical attenuation spectra of PVCP samples, arising from the combined effects of absorption and scattering. In order to simplify the inter-sample comparison, these are presented as the natural log of the proportion of the energy transmitted $T$ normalised by the sample thickness $d$. In the absence of optical scattering, this will equal the optical absorption coefficient. Fig. 2 shows the spectra which are grouped by their similarity. The intrinsic absorption peaks of PVCP can be observed at 910, 1190, 1400, 1720, 1940 and $2140 \mathrm{~nm}$ (the last two peaks are not commonly reported, possibly due to the available wavelength measurement range). For fully cured samples, the spectra is dominated by the optical absorption and does not depend on the curing temperature or time. For partially cured samples it tends to be higher, which is due to a reduced transmittance caused by the clustering of PVC particles and thus increased effect of scattering, especially 


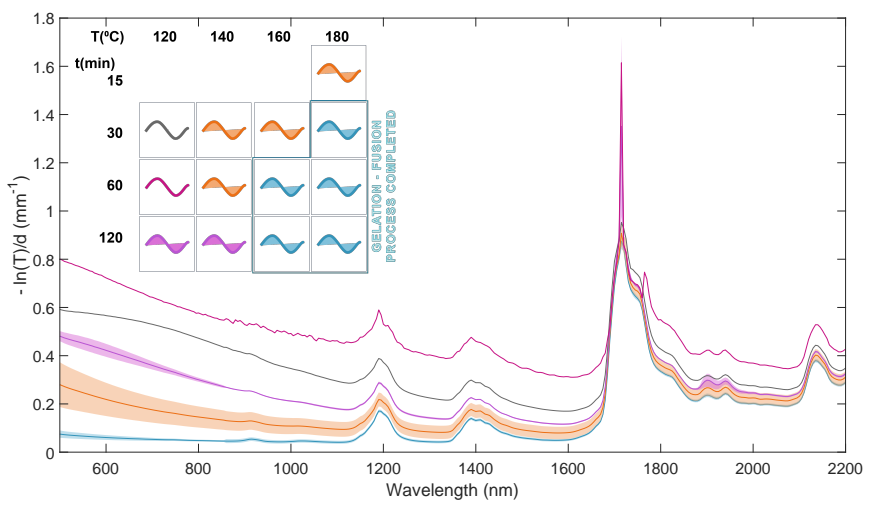

Fig. 2. The optical attenuation spectra of polyvinyl chloride plastisol (PVCP) samples made with different curing temperatures and times. The spectra are plotted as the natural $\log$ of the proportion of the energy transmitted $T$ normalised by the sample thickness $d$ over the wavelength range from $500 \mathrm{~nm}$ to $2200 \mathrm{~nm}$. In the absence of optical scattering, such as can be seen in the samples with complete gelation-fusion, this will equal the optical absorption coefficient.

prominent at shorter wavelengths. The total optical attenuation spectra of partially cured samples approaches the values for fully cured samples with increasing curing temperature and time as the optical scattering coefficient decreases to zero when PVCP has reached complete gelation-fusion.

With the addition of pigment dispersions, the intrinsic absorption peaks are less prominent as the spectrum is dominated by the colour, but are nevertheless still present. Fig. 3 shows the optical absorption spectra of PVCP samples with $0.1 \% \mathrm{v} / \mathrm{v}$ BPC, $0.1 \% \mathrm{v} / \mathrm{v}$ green pigment, and a representative spectrum of fully cured PVCP with no additives, all cured at $160^{\circ} \mathrm{C}$ for 120 minutes. The values of the total optical absorption coefficient increase as a linear function of pigment concentration [19]. For BPC, the spectra are qualitatively identical, while for other pigments, the spectrum contains additional absorption peaks characteristic of the added colour thus indicating a suitable pigment dispersion can be chosen for the wavelength of interest. It can also be observed that the addition of BPC increases the $\mu_{t}$ values more than the same concentration of the green pigment dispersion used as an exemplary colour spectrum.

\section{B. Acoustic Properties}

The effect of curing temperature and time on the acoustic properties of the PVCP samples is shown in Fig. 4 and 5. The uncertainty in the speed of sound is $3 \mathrm{~m} \cdot \mathrm{s}^{-1}$ in the whole frequency range (mostly due to temperature variations). Speed of sound (Fig. 4) increased with curing time for a given curing temperature, but not significantly, whereas the acoustic attenuation (Fig. 5) remained unchanged. The values for speed of sound are at the lower end of the range for human fatty tissue [31]. The measured frequency-dependent acoustic attenuation coefficient was fitted with a power law of the form $\alpha=\alpha_{0} f^{y}$ over the frequency range from 2 to $15 \mathrm{MHz}$, where $\alpha_{0}=0.6643 \mathrm{~dB} / \mathrm{cm} / \mathrm{MHz}^{\mathrm{y}}$ and $y=1.552\left(\mathrm{R}^{2}=0.95\right)$. The power law fit was obtained using the mean of the data for fully cured samples. These are similar to the values for normal human

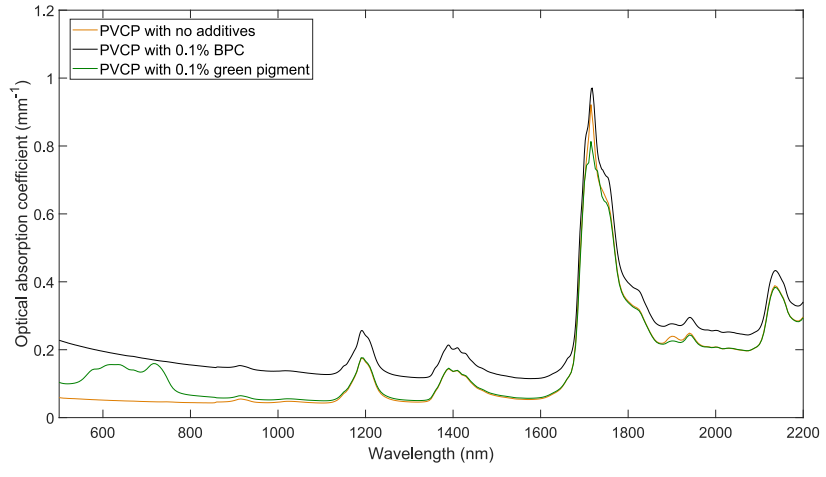

Fig. 3. The optical absorption spectra of polyvinyl chloride plastisol (PVCP) samples with pigment dispersions in comparison to the PVCP formulation with no additives.
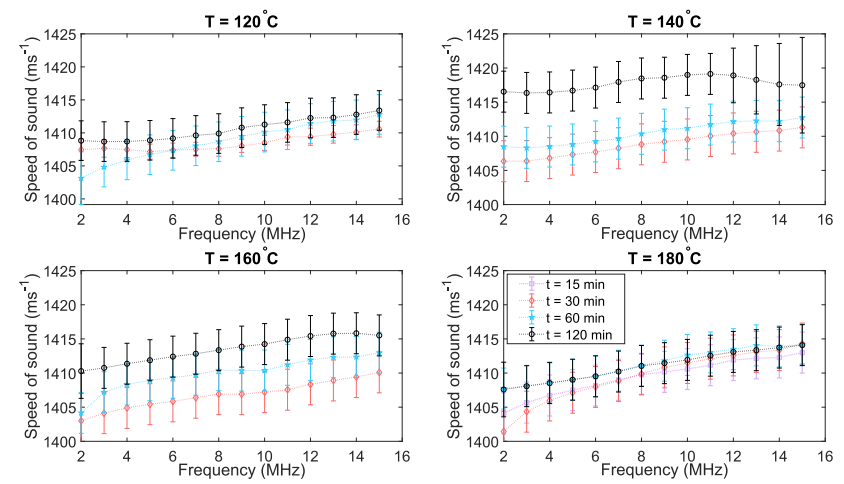

Fig. 4. The effect of curing temperature and time on polyvinyl chloride plastisol (PVCP) speed of sound at $20.0^{\circ} \mathrm{C}$ over the frequency range from $2 \mathrm{MHz}$ to $15 \mathrm{MHz}$. Error bars represent the expanded uncertainty $(\mathrm{p}=0.95)$ which equals to $3 \mathrm{~m} \cdot \mathrm{s}^{-1}$ for all the measurements.

breast tissue [31]. Expanded uncertainty was evaluated as in [26] to be $12 \%$ in the range $2-3 \mathrm{MHz}, 10 \%$ in the range $4-7$ $\mathrm{MHz}, 7 \%$ in the range $8-12 \mathrm{MHz}$, and $5 \%$ in the range $12-15$ MHz. The density of all samples was $1000 \pm 0.03 \mathrm{~kg} \cdot \mathrm{m}^{-3}$, while the acoustic impedance values for fully cured samples ranged from 1.39 to $1.51 \times 10^{6} \mathrm{~kg} /\left(\mathrm{m}^{2} \cdot \mathrm{s}\right)$ at a frequency of 5 $\mathrm{MHz}$, which is similar to values for fatty tissue and water at $20^{\circ} \mathrm{C}$ [31].

The addition of pigment dispersions of up to $1 \%$ had a very small effect on the speed of sound (up to $5 \mathrm{~m} \cdot \mathrm{s}^{-1}$ increase) and no effect on the acoustic attenuation of samples. Fig. 6 shows a comparison of the acoustic properties of PVCP samples with no additives, $0.1 \% \mathrm{BPC}$ and $1 \% \mathrm{BPC}$, all cured at $160^{\circ} \mathrm{C}$ for 120 minutes.

\section{Stability}

The samples were stored in glass containers and kept at $2{ }^{\circ} \mathrm{C}$. The statistical analysis was performed as described in [26], [32] and the normalised error was calculated over the measurement frequency range. This analysis was used to establish the significance of the observed difference between two sets of measurement data. For values of normalised 

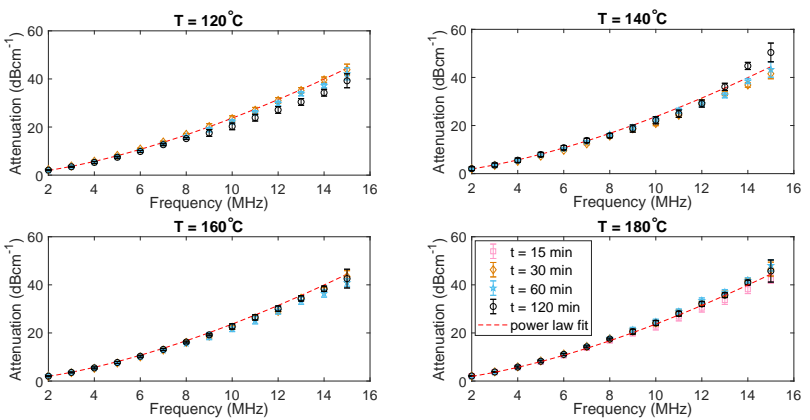

Fig. 5. The effect of curing temperature and time on polyvinyl chloride plastisol (PVCP) acoustic attenuation coefficient at $20.0^{\circ} \mathrm{C}$. Error bars represent the expanded uncertainty $(\mathrm{p}=0.95)$ over the frequency range from $2 \mathrm{MHz}$ to $15 \mathrm{MHz}$. The power law fit was obtained using the mean of the data for fully cured samples.
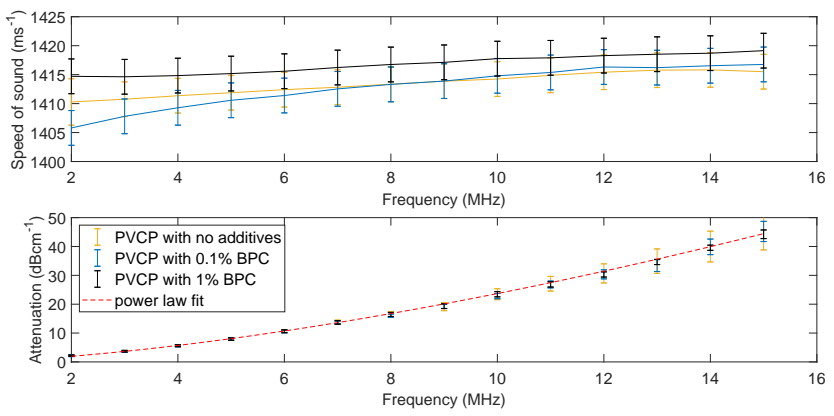

Fig. 6. Comparison of speed of sound and acoustic attenuation of polyvinyl chloride plastisol (PVCP) with no additives, and samples with $0.1 \%$ and $1 \%$ black plastic colour (BPC) at $20.0^{\circ} \mathrm{C}$. Error bars represent the expanded uncertainty $(\mathrm{p}=0.95$ ) over the frequency range from $2 \mathrm{MHz}$ to $15 \mathrm{MHz}$. The power law fit was obtained using the mean of the data for fully cured samples.

error lower than and equal to one, the compared samples were considered statistically equivalent with respect to the parameter under consideration. The observed mass and density losses over 16 weeks were less than $2 \%$ for all samples, and no significant change was observed in the measured acoustic properties. The speed of sound changed by $\pm 5 \mathrm{~m} \cdot \mathrm{s}^{-1}$, however, this was evaluated to be statistically insignificant. No change was measured for the acoustic absorption. Optical attenuation showed an increase of less than $2 \%$ for all samples.

\section{Conclusion}

The effect of curing temperature and time on PVCP properties is presented. The samples were fabricated using an optimized process in which the suspension is poured into a mould prior to curing at the high temperatures required for the completion of the gelation-fusion process, in order to avoid premature solidification and bubble formation. A simple visual method of determining the optimum temperature and time of curing was utilized by studying the colour and opacity of the cured samples which become transparent when fully cured. The density, speed of sound, acoustic attenuation and optical attenuation of the PVCP samples were measured. The stability of these properties was also assessed 16 weeks after fabrication. The results show the acoustic properties of PVCP are robust to changes in the fabrication method, and its optical properties are stable provided the material has reached complete gelation-fusion. The values of speed of sound, acoustic attenuation and acoustic impedance correspond to typical values for human fatty tissue, while the optical properties can be tuned with the addition of pigment dispersions and/or scatterers. The new fabrication method is scalable to larger volumes essential for full-size imaging phantoms used for evaluating deep-imaging photoacoustic devices.

\section{ACKNOWLEDGEMENT}

The authors would like to thank Dr Anant Shah for help with material characterisation measurements at NPL, and Dr Bob C. Schröder from the Department of Chemistry, UCL for advice on polymers and plasticizer migration.

\section{REFERENCES}

[1] D. C. Davis, R. L. McConnell, and M. F. Meyer Jr, "Poly (vinyl chloride) plastisol compositions," Aug. 30 1983, US Patent 4,401,720.

[2] H. Boudhani, C. Lainé, R. Fulchiron, and P. Cassagnau, "Rheology and gelation kinetics of PVC plastisols," Rheologica Acta, vol. 46, no. 6, pp. 825-838, 2007.

[3] N. Nakajima and C. Daniels, "Plastisols of poly (vinyl chloride); particle size distribution, morphology, rheology, and mechanism of aging," Journal of Applied Polymer Science, vol. 25, no. 9, pp. 2019-2044, 1980.

[4] J. Verdu, A. Zoller, and A. Marcilla, "Plastisol gelation and fusion rheological aspects," Journal of Applied Polymer Science, vol. 129, no. 5, pp. 2840-2847, 2013.

[5] J. A. Schey, Introduction to manufacturing processes. McGraw - Hill New York etc., 1987, vol. 2.

[6] G. Rybachuk, I. Kozlova, V. Mozzhukhin, and V. Guzeev, "PVC plastisols: Preparation, properties, and application," Polymer Science Series C, vol. 49, no. 1, pp. 6-12, 2007.

[7] P. M. Henrichs, J. Meador, K. Mehta, T. Miller, A. Yee, and A. A. Oraevsky, "Phantoms for development of LOIS as a modality for diagnostic imaging of breast cancer," in Photons Plus Ultrasound: Imaging and Sensing, vol. 5320. International Society for Optics and Photonics, 2004, pp. 8-16.

[8] G. M. Spirou, A. A. Oraevsky, I. A. Vitkin, and W. M. Whelan, "Optical and acoustic properties at $1064 \mathrm{~nm}$ of polyvinyl chloride-plastisol for use as a tissue phantom in biomedical optoacoustics," Physics in Medicine \& Biology, vol. 50, no. 14, p. N141, 2005.

[9] N. Hungr, J.-A. Long, V. Beix, and J. Troccaz, "A realistic deformable prostate phantom for multimodal imaging and needle-insertion procedures," Medical Physics, vol. 39, no. 4, pp. 2031-2041, 2012.

[10] L. Maggi, G. Cortela, M. A. von Kruger, C. Negreira, and W. C. de Albuquerque Pereira, "Ultrasonic attenuation and speed in phantoms made of PVCP and evaluation of acoustic and thermal properties of ultrasonic phantoms made of polyvinyl chloride-plastisol (PVCP)." in IWBBIO, 2013, pp. 233-241.

[11] S. E. Bohndiek, S. Bodapati, D. Van De Sompel, S.-R. Kothapalli, and S. S. Gambhir, "Development and application of stable phantoms for the evaluation of photoacoustic imaging instruments," PloS one, vol. 8, no. 9, p. e75533, 2013.

[12] O. Fenollar, L. Sanchez-Nacher, D. Garcia-Sanoguera, J. López, and R. Balart, "The effect of the curing time and temperature on final properties of flexible PVC with an epoxidized fatty acid ester as naturalbased plasticizer," Journal of Materials Science, vol. 44, no. 14, pp. 3702-3711, 2009.

[13] D. Garcia, R. Balart, F. Parres, and J. López, "Characterization of blends of poly (vinyl chloride) waste for building applications," Journal of Materials Science, vol. 42, no. 24, pp. 10 143-10151, 2007.

[14] E. Madsen, G. Frank, T. Krouskop, T. Varghese, F. Kallel, and J. Ophir, "Tissue-mimicking oil-in-gelatin dispersions for use in heterogeneous elastography phantoms," Ultrasonic Imaging, vol. 25, no. 1, pp. 17-38, 2003.

[15] A. Bykov, A. Popov, M. Kinnunen, T. Prykäri, A. Priezzhev, and R. Myllylä, "Skin phantoms with realistic vessel structure for oct measurements," in Laser Applications in Life Sciences, vol. 7376. International Society for Optics and Photonics, 2010, p. 73760F. 
[16] G. Cortela, N. Benech, W. Pereira, and C. Negreira, "Characterization of acoustical properties of a phantom for soft tissues (PVCP and graphite powder) in the range $20-45^{\circ} \mathrm{C}, "$ Physics Procedia, vol. 70, pp. 179-182, 2015.

[17] W. C. Vogt, C. Jia, B. S. Garra, and T. J. Pfefer, "Characterization of tissue-simulating polymers for photoacoustic vascular imaging," in Smart Biomedical and Physiological Sensor Technology XI, vol. 9107. International Society for Optics and Photonics, 2014, p. 910719.

[18] W. C. Vogt, C. Jia, K. A. Wear, B. S. Garra, and T. J. Pfefer, "Biologically relevant photoacoustic imaging phantoms with tunable optical and acoustic properties," Journal of Biomedical Optics, vol. 21, no. 10, pp. 101405-101 405, 2016.

[19] M. Fonseca, B. Zeqiri, P. Beard, and B. Cox, "Characterisation of a phantom for multiwavelength quantitative photoacoustic imaging," Physics in Medicine \& Biology, vol. 61, no. 13, p. 4950, 2016.

[20] R. F. Goddu and D. A. Delker, "Spectra-structure correlations for nearinfrared region," Analytical Chemistry, vol. 32, no. 1, pp. 140-141, 1960.

[21] M. B. Fonseca, L. An, and B. T. Cox, "Sulfates as chromophores for multiwavelength photoacoustic imaging phantoms," Journal of Biomedical Optics, vol. 22, no. 12, p. 125007, 2017.

[22] W. Li, B. Belmont, J. M. Greve, A. B. Manders, B. C. Downey, X. Zhang, Z. Xu, D. Guo, and A. Shih, "Polyvinyl chloride as a multimodal tissue-mimicking material with tuned mechanical and medical imaging properties," Medical Physics, vol. 43, no. 10, pp. 5577-5592, 2016.

[23] N. Nakajima, J. Isner, E. Harrell, and C. Daniels, "Dependence of gelation and fusion behavior of poly (vinyl chloride) plastisols upon particle size and size distribution," Polymer Journal, vol. 13, no. 10, p. 955, 1981.

[24] B. Zeqiri, W. Scholl, and S. P. Robinson, "Measurement and testing of the acoustic properties of materials: a review," Metrologia, vol. 47, no. 2, p. S156, 2010.

[25] S. Rajagopal, N. Sadhoo, and B. Zeqiri, "Reference characterisation of sound speed and attenuation of the IEC agar-based tissue-mimicking material up to a frequency of $60 \mathrm{MHz}$," Ultrasound in Medicine \& Biology, vol. 41, no. 1, pp. 317-333, 2015.

[26] R. M. Baêsso, R. P. Costa-Felix, P. Miloro, and B. Zeqiri, "Ultrasonic parameter measurement as a means of assessing the quality of biodiesel production," Fuel, vol. 241, pp. 155-163, 2019.

[27] United Kingdom Accreditation Service, The expression of uncertainty and confidence in measurement, 3rd ed. UKAS, 2012, vol. M3003.

[28] "JCGM 100:2008 Evaluation of measurement data - Guide to the expression of uncertainty in measurement (GUM 1995 with minor corrections)," 2008.

[29] S. Hollande and J.-L. Laurent, "Study of discolouring change in PVC, plasticizer and plasticized PVC films," Polymer Degradation and Stability, vol. 55, no. 2, pp. 141-145, 1997.

[30] A. Jimenez, J. Lopez, A. Iannoni, and J. Kenny, "Formulation and mechanical characterization of PVC plastisols based on low-toxicity additives," Journal of Applied Polymer Science, vol. 81, no. 8, pp. 18811890, 2001

[31] F. A. Duck, Physical properties of tissues: a comprehensive reference book. Academic press, 2013.

[32] A. Steele and R. Douglas, "Extending $E_{n}$ for measurement science," Metrologia, vol. 43, no. 4, p. S235, 2006.

Marina Bakaric photograph and biography not available at time of publication.

Piero Miloro photograph and biography not available at time of publication.

Bajram Zeqiri photograph and biography not available at time of publication.
Bradley E. Treeby photograph and biography not available at time of publication. 